\title{
BMJ Open Benefits and safety of transdermal glyceryl trinitrate in acute stroke: a systematic review and meta-analysis of randomised trials (protocol)
}

\author{
Beng Leong Lim (D) , ${ }^{1,2}$ Wei Feng Lee, ${ }^{1,2}$ Wei Ming Ng, ${ }^{1,3}$ Wei Ling Tay, ${ }^{1,2}$ \\ Wui Ling Chan ${ }^{1,2,4}$
}

To cite: Lim BL, Lee WF, $\mathrm{Ng}$ WM, et al. Benefits and safety of transdermal glyceryl trinitrate in acute stroke: a systematic review and meta-analysis of randomised trials (protocol). BMJ Open 2021;11:e043591. doi:10.1136/ bmjopen-2020-043591

- Prepublication history for this paper is available online. To view these files, please visit the journal online (http://dx.doi. org/10.1136/bmjopen-2020043591).

Received 09 August 2020 Revised 11 December 2020 Accepted 14 December 2020

\section{Check for updates}

\section{(c) Author(s) (or their} employer(s)) 2021. Re-use permitted under CC BY-NC. No commercial re-use. See rights and permissions. Published by BMJ.

1Department of Emergency Medicine, Ng Teng Fong General Hospital, National University Health System, Singapore

${ }^{2}$ Yong Loo Lin Medical School,

National University of Singapore, Singapore

${ }^{3}$ Unit for Prehospital Emergency Care, Government of Singapore Ministry of Health, Singapore

${ }^{4}$ Disaster Medicine, Government of Singapore Ministry of Health, Singapore

Correspondence to

Beng Leong Lim;

beng_leong_lim@nuhs.edu.sg

\section{ABSTRACT}

Introduction High blood pressure (BP) in acute stroke has adverse outcomes. Transdermal glyceryl trinitrate (GTN) has beneficial properties in controlling BP. The 2016 metaanalysis and 2017 Cochrane review showed that transdermal GTN was beneficial in a small patient subgroup with stroke onset $\leq 6$ hours. Larger studies focusing on this patient subgroup have since been conducted. We report the protocol for an updated systematic review and meta-analysis on the safety and benefits of transdermal GTN in acute stroke. Methods and analysis We will search Medline, Pubmed, Embase, CINAHL and Cochrane Library from inception until June 2020 for randomised trials that report the efficacy and safety of transdermal GTN versus placebo/control therapy among adult patients with acute stroke. Primary outcomes include in-hospital mortality, BP lowering and late functional status. Secondary outcomes include early, late, resource utilisation and surrogate outcomes. Safety outcomes include reported adverse events. Reviewers will first screen titles and abstracts, and then full texts, to identify eligible studies. Independently and in duplicate, they will extract data, assess risk of bias (RoB) using a modified Cochrane RoB tool and quality of evidence using Grading of Recommendations, Assessment, Development and Evaluation. Disagreement will be resolved by discussion and consultation with an external reviewer if necessary. Using a random-effects model, we will report effect sizes using relative risks and $95 \% \mathrm{Cls}$. We will perform predefined subgroup analyses: intracerebral haemorrhage versus ischaemic stroke; minor (NIHSS (National Institutes of Health Stroke Scale) sfive) versus major (NIHSS > five) ischaemic stroke; ischaemic stroke with versus without thrombolysis; prehospital versus non-prehospital settings; time from stroke to randomisation $\leq 6$ versus $>6$ hours and high versus low overall RoB studies. We will also perform trial sequential analysis for the primary outcomes.

Ethics and dissemination Ethics board approval is unnecessary. PROSPERO registration has been obtained. The results will be disseminated through publication in a peer-reviewed journal.

PROSPERO registration number CRD42020173093.

\section{INTRODUCTION}

High blood pressure (BP) is present in greater than $70 \%$ of patients with acute ischaemic
Strengths and limitations of study

- This is an updated meta-analysis which includes more recent larger trials.

- This study will examine an important gap on the benefits of transdermal glyceryl trinitrate (GTN) in ultra-acute stroke ( $\leq 6$ hours) identified by previous reviews.

- Other strengths include a comprehensive search strategy, an extensive predefined subgroup analysis plan and inclusion of Grading of Recommendations, Assessment, Development and Evaluation methodology to assess certainty of evidence.

- This study will be the first to use trial sequential analysis on important primary outcomes.

- Limitations include high clinical heterogeneity given the different subtypes of acute stroke, variation in timing of randomisation from onset of stroke to transdermal GTN or placebo/control therapy and reporting of outcome measures across trials.

stroke. ${ }^{1}$ It is associated with poor outcomes including acute stroke recurrence, death within a few weeks or combined death and dependency after several months. ${ }^{1-4}$ High BP is similarly common in acute intracerebral haemorrhage $(\mathrm{ICH})^{5}$ and may be associated with haematoma expansion and increased mortality. ${ }^{6-8}$

It is recommended to lower BP in $\mathrm{ICH}^{910}$ although controversy exists regarding optimal BP target in patients with ICH and there is no current literature on the role of prehospital BP reduction. The management of high BP in acute ischaemic stroke and the decision to treat or not to treat has been a constant debate since 1985. Current available guidelines recommend withholding antihypertensive therapy in the early poststroke period unless there is markedly elevated BP $(>220 / 120 \mathrm{~mm} \mathrm{Hg})$ or with BP $>185 / 110 \mathrm{~mm} \mathrm{Hg}$ for patients eligible for 
thrombolysis or BP $>180 / 105 \mathrm{~mm} \mathrm{Hg}$ during the 24-hour period following reperfusion. ${ }^{11-13}$

Nitric oxide (NO) donors are candidate agents to lower $\mathrm{BP}$ in acute stroke because NO is a cerebral and systemic vasodilator, modulates vascular and neuronal function, is neuroprotective and inhibits apoptosis. ${ }^{14}$ In addition, vascular NO concentrations are low in acute stroke which are associated with increased severity of stroke, mortality and institutionalisation. ${ }^{15}$ These observations support that NO supplementation might be beneficial.

Glyceryl trinitrate (GTN) is an example of a NO donor. Transdermal GTN administration offers a constant release of the drug across the skin into the systemic circulation for 24 hours which achieves sustained steady-state plasma concentrations. ${ }^{16}$ Transdermal GTN offers a formulation which is easily administered in many clinical settings (prehospital, Emergency Department (ED) and inpatient) managing acute stroke which may help to minimise fluctuations in drug concentrations and hence BP.

The latest meta-analysis published in $2016^{17}$ and Cochrane review in 2017, ${ }^{18}$ using data from five completed transdermal GTN trials $(n=4197)$, reported no improvement in outcomes across a range of domains, such as death, disability, cognition, mood and quality of life, with transdermal GTN versus placebo or control therapy. However, in a prespecified subgroup analysis of patients with time from stroke to randomisation $\leq 6$ versus $>6$ hours $(n=312)$, these two reviews reported a favourable functional outcome as measured by modified Rankin Scale (mRS) at 90 days with transdermal GTN. There were important limitations in these reviews. Four out of five selected trials had small sample sizes $(n \leq 90)$. The remaining multicentre ENOS trial ${ }^{19}$ recruited 4011 patients and it dominated the pooled analysis $(95.6 \%$ of all patients and $86.9 \%$ of those randomised within 6 hours of onset). In addition, all the included trials were conducted by a single research group and it is important that other research groups study the role of transdermal GTN in acute stroke. Finally, a relatively small number of patients $(n=312)$ were treated within 6 hours of stroke onset and these patients came from just two of the five trials.

The recently published multicentre RIGHT-2 trial randomised 1149 participants with acute stroke within 4 hours of onset to receive transdermal GTN versus sham therapy. ${ }^{20}$ The data from this study more than triple that used to examine the role of transdermal GTN in ultraearly stroke (onset $\leq 6$ hours). There is an urgent need to update the evidence behind the efficacy and safety of transdermal GTN in acute stroke especially among those patients with ultra-early ( $\leq 6$ hours) presentation.

The aim of this systematic review and meta-analysis is to examine, using recent data, whether transdermal GTN improves important patient-centred outcomes and is safe among patients with acute stroke in the prehospital and inhospital settings compared with placebo or control therapy by reviewing randomised controlled trials (RCTs).

\section{METHODS}

\section{Study registration}

This systematic review and meta-analysis protocol has been registered in the International Prospective Register of Systematic Reviews. We will adhere to the Preferred Reporting Items for Systematic Reviews and MetaAnalysis statement for reporting systematic review and meta-analysis. $^{21}$

\section{Eligibility criteria}

We will include randomised trials investigating the efficacy and safety of transdermal GTN versus placebo or control therapy among adult patients presenting with acute stroke.

Patients aged $\geq 16$ years presenting with either acute ischaemic or haemorrhagic stroke in the prehospital, ED and inpatient clinical settings will be considered. Acute stroke patients are defined as those with presentation within 5 days of onset of symptoms. We select 5 days since onset of symptoms as the inclusion cut-off criterion for this review because there can be significant delay in presentation after an acute stroke; especially for less severe ischaemic strokes. ${ }^{22}$ Patients with ischaemic stroke are included regardless of whether they receive thrombolysis. The comparator arms will include transdermal GTN patch, sham patch and control with existing standard therapy.

Primary outcomes are important patient-centred outcomes including in-hospital mortality, lowering of BP measurements and late functional status. BP parameters will include systolic BP, diastolic BP and mean arterial pressure measured at intervals stated by the authors. Late functional status will involve assessment using the mRS within 3 months of stroke onset or later (as reported by the authors); the preferred outcome measurement for acute stroke trials. ${ }^{23}$ The hierarchical mRS scores range from 0 to 6 , with a score of 0 indicating no symptoms, 1 indicating some symptoms but no significant disability, 2-5 indicating increasing levels of disability and dependency and 6 indicating death. ${ }^{23}$

Secondary outcomes are classified as early, late, resource utilisation and surrogate outcomes. Early secondary outcomes include development of ICH, recurrent stroke and change in calculated National Institutes of Health Stroke Scale (NIHSS). Late secondary outcomes include reported changes in activities of daily living, cognition, quality of life and mood. Resource utilisation secondary outcomes include length of hospital stay and discharge destination. Surrogate secondary outcomes include changes in cerebral haemodynamics and laboratory parameters like platelet aggregation.

Safety outcomes include any adverse events reported by the authors.

\section{Search strategy}

We will search Medline, Pubmed, Embase, CINAHL and Cochrane Library from inception until June 2020 without language restrictions. We will review reference lists for 
eligible new trials and also search ClinicalTrials.gov for ongoing or unpublished trials and for additional data from published trials. The search strategy will include the following keywords: stroke, ischaemic stroke, haemorrhagic stroke, intracerebral haemorrhage, transdermal glyceryl trinitrate, transdermal nitroglycerin, glyceryl trinitrate patch, nitroglycerin patch, trinitroglycerin, prehospital, mortality, blood pressure, functional outcome, humans and randomised clinical trials. Medical Subject Heading terms will include acute stroke, brain infarction, brain haemorrhage, prehospital emergency care, nitroglycerin, nitric oxide donors, blood pressure, haemodynamics and cerebral haemodynamics. A proposed search strategy on Medline using the Pubmed interface is attached as online supplementary appendix 1.

\section{Study selection}

Reviewers (LBL, CWL, LWF and NWM) will independently and in duplicate screen the titles and abstracts of all identified studies to generate a list of eligible trials from which full texts will be obtained. Subsequently, the same reviewers will independently assess eligibility of these full texts of published trials to decide on the final included studies. Discrepancies between reviewers will be resolved through discussion and consensus or, if needed, by adjudication from an external reviewer and/or contact with authors of the original trials for clarification.

\section{Data extraction}

Two pairs of reviewers (LBL and CWL; LWF and NWM) will extract data from included studies both independently and in duplicate. Data will be extracted using a predesigned data extraction form adapted from the Cochrane Collaboration. ${ }^{24}$ The data collection form is attached as online supplementary appendix 2. Data extracted will include the following: general study information (authors, publication year and study location(s)); study population details (clinical setting-prehospital versus ED versus inpatient, sample size, types of strokesischaemic versus haemorrhagic; subgroup of ischaemic strokes with thrombolysis); details on the comparator arms (different doses and duration of GTN patch; sham patch and control) as well as the primary, secondary and safety outcomes as listed above.

In randomised trials that included more than one arm of GTN dosing and duration, we will extract data from the arm closest to a single dose regimen that is comparable to other primary studies to be used for analysis.

Discrepancies in data extraction will be resolved through discussion and consensus or, if needed, via an external reviewer and/or contact with authors of the original trials for clarification.

\section{Risk of bias assessment}

We will assess the risk of bias (RoB) for each outcome of the individual studies using a modified Cochrane RoB instrument. ${ }^{25}$ The instrument assesses biases in the following five domains: selection bias (random sequence generation and allocation concealment); performance bias (blinding of participants and researchers); detection bias (blinding of outcome assessment); attrition bias (incomplete outcome data) and reporting bias (selective reporting). Within each domain, we will classify the RoB as high, unclear or low. Reviewers will also judge to determine whether any particular domain is impossible to achieve in any of the primary studies (like blinding in trials comparing GTN patch versus existing standard therapy) and likely or unlikely to affect the reported effect size of the outcome.

Primary studies will be classified as having an overall high RoB when they have been rated at least one domain as having high risk after exclusion of certain domain that is judged to be logistically impossible to achieve for that particular trial and unlikely to affect reported effect size of outcome. The overall RoB for each individual trial will be considered low if RoB is judged to be low in all domains and unclear if RoB is judged to be unclear in any of the domains.

\section{Quality of evidence}

We will also assess the quality of evidence for each outcome using the GRADE (Grading of Recommendations, Assessment, Development and Evaluation) approach that classifies evidence as high, moderate, low or very low quality based on considerations of RoB, consistency, directness, precision and publication bias. ${ }^{26}$ We attach a summary of findings table (online supplementary appendix 3) which is adapted using the GRADEpro software to demonstrate how we will present our GRADE assessment for the main outcomes.

Assessment of the individual and overall RoB categories as well as the quality of evidence will be performed independently by the two pairs of reviewers (LBL and CWL; LWF and NWM) with any discrepancies resolved by discussion and consensus or if necessary, via consultation with an external reviewer.

\section{Data analysis}

All analyses will be performed using RevMan V.5.3 (Cochrane Collaboration, Oxford) software. We will use DerSimonian and Laird random-effects model a priori to conduct the data analysis and meta-analysis. We chose the random-effects model as it produces more conservative CIs and it considers both within-study and between-study variability. ${ }^{21}$

For continuous outcomes, we will calculate the mean difference and its corresponding 95\% CIs whenever possible. For dichotomous outcomes, we will calculate the relative risk and its corresponding 95\% CIs. We will generate forest plots to demonstrate the individual and pooled effect sizes for the outcome of interest if there are at least two studies. We will assess for heterogeneity between studies by first visual inspection of the forest plots and then using the $I^{2}$ statistic. $I^{2}$ measures the percentage of the total variation in estimated effects of the outcome across studies that is due to heterogeneity rather than to 
chance. ${ }^{27} \mathrm{~A} I^{2}$ value of $0 \%$ indicates no observed heterogeneity, and larger values show increasing heterogeneity.

Regardless of the observed statistical heterogeneity $\left(I^{2}\right.$ values), we plan to conduct the following a priori subgroup analyses for each outcome when each subgroup is represented by at least two studies. These subgroup analyses will be: ICH versus ischaemic stroke; minor (NIHSS $\leq$ five) versus major (NIHSS >five) ischaemic stroke; ischaemic stroke with versus without thrombolysis; prehospital versus non-prehospital (ED and inpatient) settings; time from stroke to randomisation $\leq 6$ versus $>6$ hours; time from stroke to randomisation $\leq$ two versus $>$ two hours and high versus low overall RoB studies.

Missing data in the primary studies will be addressed in several ways. We will evaluate for rates of missing data in these primary studies, reasons for missing data and to contact primary authors for clarification if necessary. We will determine whether authors of these primary studies attempted to address the impact of missing data by using intention-to-treat analysis and performing sensitivity analyses through methods like imputation, best-case and worst-case scenario analyses to investigate how their reported effect size estimates had changed. We will then make judgement independently, through consensus and/ or consultation with an external reviewer whether the reported effect size estimates (including any sensitivity analyses) by the primary authors will likely or unlikely be affected by their missing data. We will perform separate sensitivity analyses of our pooled results by including and excluding those studies that are judged likely to be affected by missing data to investigate how the pooled effect size estimates will be affected. Finally, we will also assess the risk of missing data (attrition bias) of the primary studies through our RoB and GRADE assessments.

Meta-analyses may result in type I errors due to an increased risk of random error when sparse data are collected and repeated significance testing when a cumulative meta-analysis is updated with new trials. ${ }^{28}{ }^{29}$ We will perform trial sequential analysis (TSA) using a randomeffects model for the primary outcomes (in-hospital mortality, BP lowering and late functional status). In the TSA, we will use a statistical significance level of $5 \%$, a power of $80 \%$ and an estimated effect size difference (or mean difference for continuous outcomes) between transdermal GTN versus placebo or control therapy as reported by the included trials. TSA generates the required information size calculated as diversity-adjusted information size (DIS $)^{30}$ suggested by the estimated effect size difference; thereby providing important information on how many more patients need to be included in further trials. TSA also creates adjusted thresholds for statistical significance (trial sequential monitoring boundaries) with addition of each new trial. ${ }^{28} 29$ The cumulative $\mathrm{Z}$ curve which includes the selected trials; if it crosses the trial sequential monitoring boundary, will signify that a sufficient level of evidence has been reached and no further trials are needed. ${ }^{28}{ }^{29}$ If the $\mathrm{Z}$ curve fails to cross the trial sequential monitoring boundary, the required information size is not reached and there is insufficient evidence to reach a conclusion.

TSA will be performed using TSA V.0.9.5.10 beta (Copenhagen Trial Unit, Centre for Clinical Intervention Research, Rigshospitalet, Copenhagen, Denmark, www. ctu.dk/tsa).

\section{PATIENT AND PUBLIC INVOLVEMENT}

We have not and will not involve new patients or the public in this protocol.

\section{DISCUSSION}

Acute ischaemic and haemorrhagic strokes are frequently managed in various clinical settings; from prehospital, ED to inpatient. High BP is common in both types of strokes and is associated with short-term poor outcomes (acute stroke recurrence, death within a few weeks ${ }^{1-4}$ and haematoma expansion ${ }^{6-8}$ and adverse effects in the longer term (delayed death and dependency after several months).${ }^{1-4} \mathrm{BP}$ control is an essential part of the management of acute ischaemic and haemorrhagic strokes.

NO donors are candidate agents to lower BP in acute stroke because of its various beneficial properties ranging from vasodilatation to neuroprotection and inhibition of apoptosis. ${ }^{14}$ GTN is an example of a NO donor. Transdermal GTN offers an easily administered formulation which is valuable especially in the prehospital and ED settings to provide constant drug release.

There was a meta-analysis in $2016^{17}$ and Cochrane review in $2017^{18}$ that investigated the effects of transdermal GTN in acute stroke which reported no overall benefits. However, they reported a favourable functional outcome (improvement in mRS at 90 days) with transdermal GTN versus placebo or control therapy in a prespecified subgroup of patients with ultra-acute stroke (time from stroke to randomisation $\leq 6$ hours). The metaanalysis and Cochrane review had important limitations. Apart from the ENOS trial, ${ }^{19}$ the remaining four included trials had small sample sizes $(\mathrm{n} \leq 90)$ and all these trials were conducted by a single research group. In addition, that subgroup analysis involving ultra-acute stroke patients also suffered from a small sample size $(n=312)$.

With the inclusion of the recently published multicentre RIGHT-2 trial which recruited 1149 patients with acute stroke within 4 hours of onset, ${ }^{20}$ our systematic review and meta-analysis will significantly increase the sample size available for pooling of studies; especially so when it will more than triple that used to examine the role of transdermal GTN in ultra-acute stroke (onset $\leq 6$ hours). Our planned subgroup analysis of patients with ultra-acute stroke will address a significant gap in the literature that arose from these previous reviews.

In addition, our TSA for the important primary outcomes will reduce type I error. Our TSA will determine whether the DIS and trial sequential monitoring boundaries for these outcomes have indeed been reached in our 
meta-analysis; signifying that a sufficient level of evidence has been attained to reach a conclusion.

Other strengths of our protocol include a comprehensive search strategy of published and unpublished literature, extensive subgroup analyses involving clinically important patient subgroups and using GRADE methodology to assess certainty of evidence.

Limitations to our protocol include the anticipated high clinical heterogeneity given the haemorrhagic and ischaemic subtypes of acute stroke as well as variation in timing of randomisation from stroke onset to transdermal GTN or placebo/control therapy and reporting of outcome measures across trials even within a subtype of acute stroke. We will address clinical heterogeneity by evaluating for statistical heterogeneity, explore predefined clinically important subgroup analyses and to account for inconsistencies in our GRADE evaluation. In order to address for differences in reporting of outcome measures across included trials, we will include a spectrum of primary and secondary outcomes. We will assess reporting of these outcomes independently and in duplicate and if there are discrepancies, we will resolve through discussion, consensus, potentially involving an external reviewer and contacting the primary authors for clarification.

In conclusion, this protocol describes the details and methodology of a planned systematic review and metaanalysis addressing the safety and benefits of transdermal GTN in acute stroke. The results of this meta-analysis are expected to fill the gap in the literature on the subgroup of patients with ultra-acute stroke (onset $\leq 6$ hours), inform daily practice, clinical practice guidelines and guide areas of investigation for future RCTs.

Correction notice This article has been corrected since it first published. The provenance and peer review statement has been included.

Acknowledgements However, we wish to thank the research program of $\mathrm{Ng}$ Teng Fong Hospital Emergency Department which provided some protected time for us to carry out this work.

Contributors LBL, CWL and LWF conceived the study. LBL, CWL and LWF also wrote the study protocol to be registered with PROSPERO. All authors (LBL, CWL, LWF, NWM and TWL) contributed to protocol development. LBL, CWL and LWF drafted the study protocol and this manuscript. All authors (LBL, CWL, LWF, NWM and TWL) contributed to refinement of the study protocol and manuscript as well as approved the final manuscript.

Funding The authors have not declared a specific grant for this research from any funding agency in the public, commercial or not-for-profit sectors.

Competing interests None declared.

Patient consent for publication Not required.

Provenance and peer review Not commissioned; externally peer reviewed.

Data availability statement Data are available upon reasonable request.

Supplemental material This content has been supplied by the author(s). It has not been vetted by BMJ Publishing Group Limited (BMJ) and may not have been peer-reviewed. Any opinions or recommendations discussed are solely those of the author(s) and are not endorsed by BMJ. BMJ disclaims all liability and responsibility arising from any reliance placed on the content. Where the content includes any translated material, BMJ does not warrant the accuracy and reliability of the translations (including but not limited to local regulations, clinical guidelines, terminology, drug names and drug dosages), and is not responsible for any error and/or omissions arising from translation and adaptation or otherwise.
Open access This is an open access article distributed in accordance with the Creative Commons Attribution Non Commercial (CC BY-NC 4.0) license, which permits others to distribute, remix, adapt, build upon this work non-commercially, and license their derivative works on different terms, provided the original work is properly cited, appropriate credit is given, any changes made indicated, and the use is non-commercial. See: http://creativecommons.org/licenses/by-nc/4.0/.

ORCID iD

Beng Leong Lim http://orcid.org/0000-0002-5380-4905

\section{REFERENCES}

1 Leonardi-Bee J, Bath PMW, Phillips SJ, et al. Blood pressure and clinical outcomes in the International stroke trial. Stroke 2002;33:1315-20.

2 Willmot M, Leonardi-Bee J, Bath PMW. High blood pressure in acute stroke and subsequent outcome: a systematic review. Hypertension 2004;43:18-24.

3 Sprigg N, Gray LJ, Bath PMW, et al. Relationship between outcome and baseline blood pressure and other haemodynamic measures in acute ischaemic stroke: data from the TAIST trial. $J$ Hypertens 2006;24:1413-7.

4 Geeganage C, Tracy M, England T, et al. Relationship between baseline blood pressure parameters (including mean pressure, pulse pressure, and variability) and early outcome after stroke: data from the Tinzaparin in acute ischaemic stroke trial (TAIST). Stroke 2011;42:491-3

5 Qureshi Al, Ezzeddine MA, Nasar A, et al. Prevalence of elevated blood pressure in 563,704 adult patients with stroke presenting to the ED in the United States. Am J Emerg Med 2007;25:32-8.

6 Qureshi Al. The importance of acute hypertensive response in $\mathrm{ICH}$. Stroke 2013;44:S67-9.

7 Dandapani BK, Suzuki S, Kelley RE, et al. Relation between blood pressure and outcome in intracerebral hemorrhage. Stroke 1995;26:21-4.

8 Anderson CS, Huang $\mathrm{Y}$, Arima $\mathrm{H}$, et al. Effects of early intensive blood pressure-lowering treatment on the growth of hematoma and perihematomal edema in acute intracerebral hemorrhage: the intensive blood pressure reduction in acute cerebral haemorrhage trial (interact). Stroke 2010;41:307-12.

9 Intercollegiate Stroke Working Party. National clinical guideline for stroke. 5th edn. London: Royal College of Physicians, 2016.

10 Hemphill JC, Greenberg SM, Anderson CS. American heart association stroke Council; Council on cardiovascular and stroke nursing; Council on clinical cardiology. guidelines for the management of spontaneous intracerebral hemorrhage: a guideline for healthcare professionals from the American heart Association/ American stroke association. Stroke 2015;46:2032-60.

11 Jauch EC, Saver JL, Adams HP, et al. Guidelines for the early management of patients with acute ischemic stroke: a guideline for healthcare professionals from the American heart Association/ American stroke association. Stroke 2013;44:870-947.

12 Powers WJ, Rabinstein AA, Ackerson T. Guidelines for the early management of patients with acute ischemic stroke: a guideline for healthcare professionals from the American heart Association/ American stroke association. Stroke 2018;2018:e46-110.

13 Shinohara Y, Yamaguchi T. Outline of the Japanese guidelines for the management of stroke 2004 and subsequent revision. Int J Stroke 2008;3:55-62.

14 Willmot MR, Bath PMW. The potential of nitric oxide therapeutics in stroke. Expert Opin Investig Drugs 2003;12:455-70.

15 Rashid PA, Whitehurst A, Lawson N, et al. Plasma nitric oxide (nitrate/nitrite) levels in acute stroke and their relationship with severity and outcome. J Stroke Cerebrovasc Dis 2003;12:82-7.

16 Todd PA, Goa KL, Langtry HD. Transdermal nitroglycerin (glyceryl trinitrate). A review of its pharmacology and therapeutic use. Drugs 1990;40:880-902.

17 Bath PM, Woodhouse L, Krishnan K. Effect of treatment delay, stroke type, and thrombolysis on the effect of glyceryl trinitrate, a nitric oxide donor, on outcome after acute stroke: a systematic review and meta-analysis of individual patient from randomised trials. Stroke Res Treat 2016;2016:9706720.

18 Bath PM, Krishnan K, Appleton JP. Nitric oxide donors (nitrates), L-arginine, or nitric oxide synthase inhibitors for acute stroke. Cochrane Database Syst Rev 2017;4:CD000398.

19 Bath PM, Woodhouse L, Scutt P, et al. Efficacy of nitric oxide, with or without continuing antihypertensive treatment, for management of high blood pressure in acute stroke (eNOS): a partial-factorial randomised controlled trial. Lancet 2015;385:617-28. 
20 Bath PM, Scutt P, Anderson CS, et al. Prehospital transdermal glyceryl trinitrate in patients with ultra-acute presumed stroke (RIGHT-2): an ambulance-based, randomised, sham-controlled, blinded, phase 3 trial. Lancet 2019;393:1009-20.

21 Liberati A, Altman DG, Tetzlaff $\mathrm{J}$, et al. The PRISMA statement for reporting systematic reviews and meta-analyses of studies that evaluate healthcare interventions: explanation and elaboration. BMJ 2009;339:b2700

22 Addo J, Ayis S, Leon J, et al. Delay in presentation after an acute stroke in a multiethnic population in South London: the South London stroke register. J Am Heart Assoc 2012;1:e001685.

23 Lees KR, Bath PMW, Schellinger PD, et al. Contemporary outcome measures in acute stroke research: choice of primary outcome measure. Stroke 2012;43:1163-70.

24 Cochrane Effective Practice and Organisation of Care (EPOC). Data collection form. EPOC resources for review authors, 2017. Available: epoc.cochrane.org/resources/epoc-specific-resources-reviewauthors [Accessed 22 Nov 2020].
25 Higgins JPT, Altman DG, Gøtzsche PC, et al. The Cochrane collaboration's tool for assessing risk of bias in randomised trials. BMJ 2011;343:d5928.

26 Guyatt GH, Oxman AD, Vist GE, et al. Grade: an emerging consensus on rating quality of evidence and strength of recommendations. BMJ 2008;336:924-6.

27 Higgins JPT, Thompson SG, Deeks JJ, et al. Measuring inconsistency in meta-analyses. BMJ 2003;327:557-60.

28 Wetterslev J, Thorlund K, Brok J, et al. Trial sequential analysis may establish when firm evidence is reached in cumulative meta-analysis. $\mathrm{J}$ Clin Epidemiol 2008;61:64-75

29 Brok J, Thorlund K, Wetterslev J, et al. Apparently conclusive metaanalyses may be inconclusive--Trial sequential analysis adjustment of random error risk due to repetitive testing of accumulating data in apparently conclusive neonatal meta-analyses. Int $\mathrm{J}$ Epidemiol 2009;38:287-98

30 Wetterslev J, Thorlund K, Brok J, et al. Estimating required information size by quantifying diversity in random-effects model meta-analyses. BMC Med Res Methodol 2009;9:86. 